\title{
Uso del silicio poroso funcionalizado con acetilcolinesterasa como plataforma de detección de arsénico (III)
}

\author{
Use of porous silicon functionalized with acetylcholinesterase as detection platform \\ for arsenic (III) \\ Balderas-Valadez Ruth Fabiola \\ Universidad Autonóma del Estado de Morelos \\ Centro de Investigación en Ingeniería y Ciencias Aplicadas \\ Correo: ruth.balderas@uaem.mx \\ Agarwal Vivechana \\ Universidad Autónoma del Estado de Morelos \\ Centro de Investigación en Ingeniería y Ciencias Aplicadas \\ Correo: vagarwal@uaem.mx
}

\section{Resumen}

Sustratos nanoestructurados de silicio poroso funcionalizados con Acetilcolinesterasa (AChE) de dos diferentes diámetros de poros (muestras circulares de $1.2 \mathrm{~cm}$ de diámetro), se evaluaron como plataforma de detección de arsénico (III). La inhibición de la actividad enzimática del AChE en presencia de As (III) se usó para generar especificidad en el sensor óptico de As propuesto. Previo al enlazamiento del AChE la superficie de silicio poroso se modificó a través de 3-aminopropil (dietoxi)-metilsilano, seguida de gluteraldehido y la proteína. Cada paso de la funcionalización se caracterizó por espectroscopia de UV-vis-NIR acoplada al accesorio de reflectancia especular para monitorear el incremento del espesor óptico debido a la infiltración de las moléculas. La espectroscopia de FTIR mostró la presencia de enlaces amida I y II debidos al enlazamiento del AChE con el substrato de SiP. Un arreglo enzimático basado en el reactivo de Ellman se empleó para seguir la actividad enzimática de la AChE inmovilizada sobre SiP, así como su inhibición en presencia de As (III). El sistema de detección del progreso de la reacción se basó en el cambio del espesor óptico efectivo de película revelado como el desplazamiento del espectro de reflectancias (método referido como espectroscopia con transformada de Fourier del interferómetro de reflectancia). La respuesta óptica de las muestras de SiP modificadas en presencia de As (III) se comparó con la muestra de control y reveló un límite de detección de $0.77 \mu \mathrm{M}$.

Descriptores: Sensor, detección de arsénico, silicio poroso, RIFTS.

\begin{abstract}
Acetylcholinesterase (AChE) functionalized nanostructured porous silicon substrates with two different pore diameters (circular samples with $1.2 \mathrm{~cm}$ of diameter), have been evaluated as detection platforms for arsenic (III). Inhibition of enzymatic activity of AChE in the presence of As (III) was used to generate specificity in the proposed silicon based optical As sensors. Prior to AChE attachment, oxidized porous silicon surface was modified through 3-aminopropyl (diethoxy) -methylsilane binding followed by glutaraldehyde and the protein. Each functionalization step was characterized by UV-vis-NIR spectrophotometry coupled with the specular reflectance accessory to monitor the increase in optical thickness due to the infiltration of the molecules. FTIR spectroscopy showed the presence of amide I and II bonds due to the covalent binding of acetylcholinesterase with porous silicon substrate. An enzyme assay based on Ellman's reagent was used to test the enzymatic activity of acetylcholinesterase immobilized on porous silicon as well as its inhibition in the presence of As (III). The detection system of the reaction progress was based on the change in the effective optical thickness of the film revealed as a shift in the reflectivity spectra (method referred as reflective interferometric Fourier transform spectroscopy). The optical response of the modified substrates in the presence of As (III) was compared with the control sample and revealed the detection limit of $0.77 \mu \mathrm{M}$.
\end{abstract}

Keywords: Sensor, detection of arsenic, porous silicon, RIFTS. 


\section{INTRODUCCIÓN}

El desarrollo de sensores específicos para la detección de metales "suaves" y "pesados", es una continua meta en el campo de remediación ambiental debido al alto riesgo que estos representan a la salud ( $\mathrm{Li}$ et al., 2001; Salomons et al., 2012). El As (III) se asocia a cáncer de hígado, pulmones, pie, riñón, páncreas y próstata (Whitacre, 2012). La presencia de As se puede determinar por algunos métodos de detección como espectroscopia de absorción atómica (Wang et al., 2016; Zhuravlev et al., 2015), plasma inductivamente acoplado a espectroscopia óptica de emisión y espectroscopia de masas (Liu et al., 2015; Maher et al., 2015). Sin embargo, estas técnicas requieren condiciones específicas en el laboratorio y son consumidoras de tiempo. En la última década se han propuesto una gran variedad de métodos de detección de As que no requieren equipos especializados, van desde el uso de ensayos colorimétricos basados en bacterias (Diesel et al., 2009; De Mora et al., 2011), la modificación y mejora de superficies con nanopartículas de plata funcionalizadas $\mathrm{FeOOH}$ para usar SERS (Pradhan et al., 2015), o el uso de métodos electroquímicos con electrodos funcionalizados con agentes biológicos (Sarkar et al., 2010). Por ejemplo, Sanllorete et al. (2010) utilizaron un electrodo decorado con acetilcolinesterasa (AChE) para la detección amperométrica de As (III). Esta detección se basa en la inhibición de la actividad enzimática de AChE generada por los iones de As (III) (Stoytcheva et al., 1998). La AChE es una enzima que hidroliza el neurotransmisor Acetilcolina (ecuación 1) en las uniones neuromusculares y en otras sinapsis colinérgicas de interrumpir la señal neuronal. La inhibición de esta enzima se estudia como tratamiento del Parkinson y Alzheimer (Aarsland et al., 2004; Moss et al., 2016).

Acetilcolina iodida $+\mathrm{H}_{2} \mathrm{O} \stackrel{\text { AChE }}{\rightarrow}$ Tiocolina iodida + Ácido acético

La actividad enzimática de la AChE usualmente se analiza mediante espectroscopia UV-Vis utilizando el reactivo de Ellman et al. (1961), ditiolbisnitrovenzoato (DTNB), que reacciona con el producto de la hidrolisis de la acetilcolina para generar un producto colorido según:

Tiocolina iodida + DTNB $\rightarrow$ Producto amarillo

Por otro lado, el silicio poroso (SiP) es un material inorgánico nano-estructurado, de alta área superficial formado por la corrosión de silicio cristalino en presencia de ácido fluorhídrico donde la porosidad, el espesor de la película y el tamaño de poro se controlan por los parámetros de fabricación. Este posee una gran flexibilidad para la fabricación de diversos dispositivos ópticos (Jane et al., 2009), debido a que su estructura y propiedades ópticas (índice de refracción y absortividad) pueden manipularse a través de las condiciones de preparación de este material. Las estructuras más complejas fabricadas a base de SiP tienen como unidad fundamental la monocapa. Esta se produce cuando se suministra al sistema electroquímico una densidad de corriente fija en un cierto tiempo. Por sí sola, la monocapa podría considerarse como un dispositivo óptico débil, pues posee una baja reflectividad, respecto a otras estructuras más complejas. El espectro de reflectancia de una monocapa (\%R vs $\lambda$ ) se compone de ondulaciones sinusoidales, debido a la formación de ondas de interferencia constructivas y destructivas al interior de los poros (Vincent, 1998; Jane et al., 2009; Balderas et al., 2014).

Las estructuras de $\mathrm{SiP}$ se han estudiado como plataforma de detección óptica, basándose en el desplazamiento que el espectro de reflectancia sufre cuando se cambia el índice de refracción efectivo de la estructura, lo que se provoca por la introducción de algún analito determinado a los poros del material. La magnitud del desplazamiento del espectro para una dosis dada de analito depende de 3 factores:

a) El tamaño del analito.

b) El grado en el cual en analito incrementa el índice de refracción de la solución buffer.

c) La afinidad de la superficie de SiP con el analito (Pacholski et al., 2005).

En una monocapa de SiP el desplazamiento comúnmente es cuantificado usando la diferencia del Espesor Óptico Efectivo (EOE) antes y después de algún evento (introducción de analito, cambio de medio, etcétera). El EOE se define como 2 veces el índice de refracción $(n)$ por el espesor físico de la capa $(d)$ y se puede calcular al aplicar la transformada rápida de Fourier (FFT) al espectro de reflectancia representado como $\% \mathrm{R}$ vs $1 / \lambda$, lo que genera un pico característico, donde el punto más alto coincide con el EOE y la amplitud de pico $(A)$ está relacionada con el índice de refracción del medio poroso y el medio que lo rodea (Orosco et al., 2009). Derivado del anterior procedimiento se desarrolló la espectroscopia de interferometría de reflectancia con transformada de Fourier (RIFTS) (Pacholski et al., 2006 y 2009), que consiste en el análisis en tiempo real del cambio del EOE o la $A$, debido a la introducción de analitos específicos que interactúan con la superficie 
del SiP generando una señal que se identifica fácilmente mediante los gráficos de $\Delta \mathrm{EOE}$ vs t ó $\Delta A$ vs t. Esta técnica ha permitido detectar reacciones enzimáticas, separación por tamizado molecular y biomoleculas (Pacholski et al., 2005; Orosco et al., 2009; Pacholski et al., 2006 y 2009).

Shtenberg et al. (2015) desarrollaron una plataforma de biosensado en una película delgada tipo Fabri-Perot de SiP, que permitía el monitoreo de metales pesados en solución acuosa mediante la inhibición de la actividad enzimática de la enzima peroxidasa inmovilzada en la película porosa.

En este trabajo de investigación se propone usar el SiP funcionalizado con la AChE como superficie transductora para registrar la influencia de la presencia de As (III) en la actividad enzimática. Se utilizará la espectroscopia con transformada de Fourier del interferómetro de reflectancia (RIFTS) para registrar la actividad enzimática de las muestras de SiP funcionalizadas con $\mathrm{AChE}$ (que se referirán más adelante como: SiP/AChE). El monitoreo se realizó en tiempo real usando una celda de flujo como reactor del ensayo enzimático.

\section{Metodología eXPerimental}

Se fabricaron monocapas de SiP de dos distintos diámetros de poros por ataque electroquímico, se empleó una solución electrolítica de HF (48\%), etanol y glicerol en una relación volumétrica de 3:7:1. Se aplicó una corriente de $55 \mathrm{~mA}$ por $4 \mathrm{~min}$ y otra de $65 \mathrm{~mA}$ por $3 \mathrm{~min}$ para generar dos tipos de muestras. Se utilizó una oblea de silicio tipo $\mathrm{p}^{++}$con orientación cristalina (100) y resistivi- dad de $\rho=0.001-0.005 \Omega \mathrm{cm}$. Las muestras de SiP se oxidaron térmicamente a $600^{\circ} \mathrm{C}$ por 1 hora, con el fin de estabilizar y preparar las superficies para una subsecuente aminosilanización. La oxidación confiere una superficie hidrofílica que permite la penetración de soluciones acuosas. Las muestras de $\mathrm{SiP}$ se sumergieron durante 60 minutos en una solución a 5\% de 3-aminopropil (dietoxi)-metilsilano (APDEMS) en tolueno (este proceso proporcionó una terminación superficial del SiP con grupos amino $\left(\mathrm{NH}_{2}\right)$ ). Para eliminar los residuos de APDEMS la muestra se lavó en forma consecutiva con tolueno y una solución de agua/etanol (1:1) y se secó con un flujo suave de $\mathrm{N}_{2}$. Posteriormente y para asegurar la eliminación del solvente orgánico y promover la polimerización horizontal del APDEMS sobre el $\mathrm{SiP}$, la muestra se horneó a $110^{\circ} \mathrm{C}$ durante $15 \mathrm{~min}$. Después las estructuras de SiP modificadas con APDEMS (SiP/APDEMS) se sumergieron en una solución de glutaraldehído (GTA) a 2.5\% diluido en PBS 10 mM, pH 8, por $90 \mathrm{~min}$. Los enlaces no específicos se eliminaron lavando con PBS y secando las muestras con flujo suave de $\mathrm{N}_{2}$. Finalmente, las muestras modificadas SiP/APDEMS/GTA se incubaron durante 12 hrs en soluciones de acetilcolinesterasa diluida en PBS $10 \mathrm{mM} \mathrm{pH} \mathrm{8,} \mathrm{a} \mathrm{di-}$ ferentes concentraciones $(0.1 \mathrm{mg} / \mathrm{mL}$ y $0.2 \mathrm{mg} / \mathrm{mL})$, el proceso se realizó en refrigeración. La enzima no unida covalentemente a la superficie se removió lavando las estructuras con PBS y se secaron con un flujo suave de $\mathrm{N}_{2}$. Las muestras de $\mathrm{SiP} / \mathrm{AChE}$ se almacenaron en cajas de petri plásticas previamente marcadas y selladas con parafilm, y se refrigeraron a $4^{\circ} \mathrm{C}$ hasta su uso. La figura 1

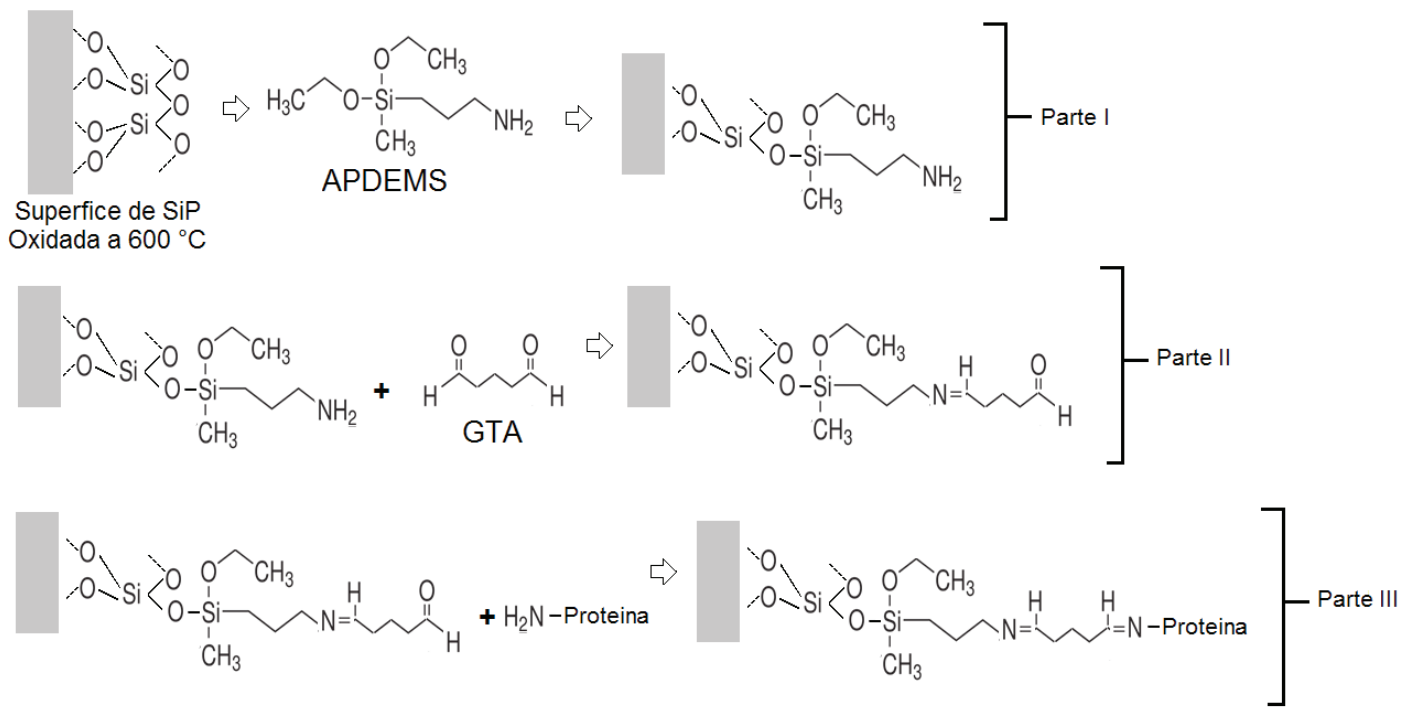

Figura 1. Esquema del procesos de funcionalización de la superficie de SiP con AChE vía enlaces amidas 
muestra un diagrama esquemático de las reacciones aplicadas en esta funcionalización.

Cada paso del proceso de funcionalización de la superficie de SiP se monitoreó mediante el registro de los cambios en el espectro de reflectancia especular de la capa porosa usando espectrofotometría UV-Vis-NIR, acoplado al accesorio de reflectancia especular con un barrido en el rango de 400 a $2500 \mathrm{~nm}$.

$\mathrm{Al}$ espectro de refletancia presentado como \% $\mathrm{R}$ vs $(1 / \lambda)$ se le aplicó FFT para obtener directamente el valor de $2 n L$ que se refiere como EOE de la capa porosa, lo que se aplicó como medida de comparación para la detección de la infiltración y anclaje de moléculas (APDEMS, GTA y AChE). La formación de enlaces covalentes se comprobaron caracterizando mediante espectrometría FTIR.

Se prepararon dos soluciones reactivas basadas en el protocolo descrito por Ellman et al. (1961) para el análisis de la actividad de la AChE por método de absorbancia. La solución 1 se empleó para comprobar la actividad enzimática del SiP/AChE y contenía acetilcolina iodide $0.3 \mathrm{mM}$ y DTNB $0.2 \mathrm{mM}$ en buffer de PBS 10 $\mathrm{mM}$. La solución 2 que se empleó para registrar la inhibición de la AChE en presencia de As (III), contenía ace-

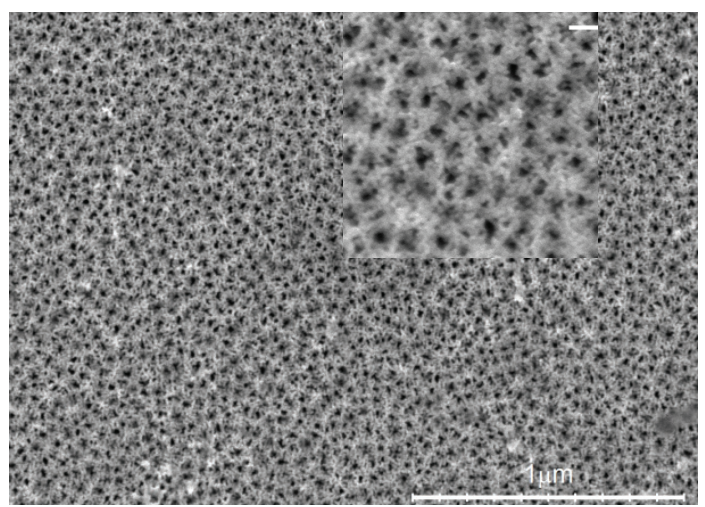

a)

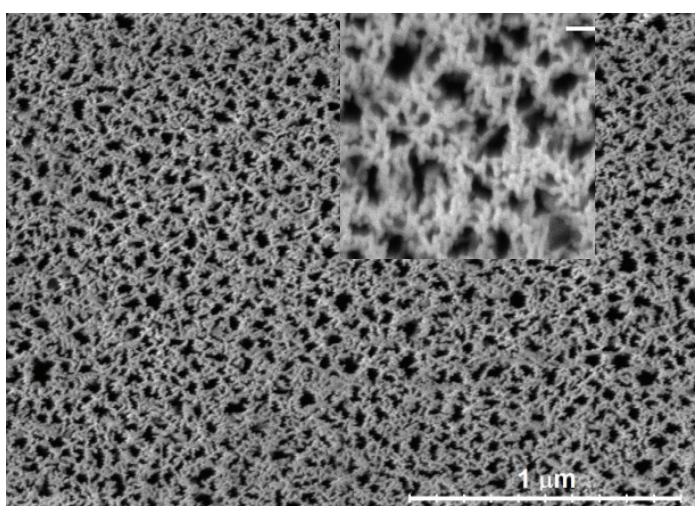

C) tilcolina iodide $0.6 \mathrm{mM}$ y DTNB $0.4 \mathrm{mM}$ en buffer de PBS $10 \mathrm{mM}$. El SiP/AChE se expone a la solución reactiva dentro de una celda de flujo conectada a una bomba de recirculación a un flujo de $5 \mu \mathrm{l} / \mathrm{min}$. Se registró el espectro de reflectancia en tiempo real usando un espectrofotómetro de fibra óptica (Ocean Optics HR 4000) colocado sobre la celda de flujo, donde se recirculó en ella solución para el ensayo enzimático agregándole Arsenito de sodio $\left(\mathrm{NaAsO}_{2}\right)$ a diferentes concentraciones. La FFT del espectro de reflectancia se calculó automáticamente utilizando el software IGOR Pro (www. wavemetrics.com) (Sailor, 2012).

\section{ResUltados Y DISCUSIÓN}

\section{MiCROSCOPIA ELECTRÓNICA DE BARRIDO}

La figura 2 muestra la micrografía de la parte superior y la sección transversal de las muestras de SiP fabricadas a diferentes corrientes (FESEM Hitachi S5500). Asimismo, la figura 2a muestra la vista superior de una monocapa sintetizada a $55 \mathrm{~mA}$, donde se observa un tamaño de poro de $16.1376 \pm 5.7246 \mathrm{~nm}$ de diámetro, relativamente menor a la monocapa sintetizada con
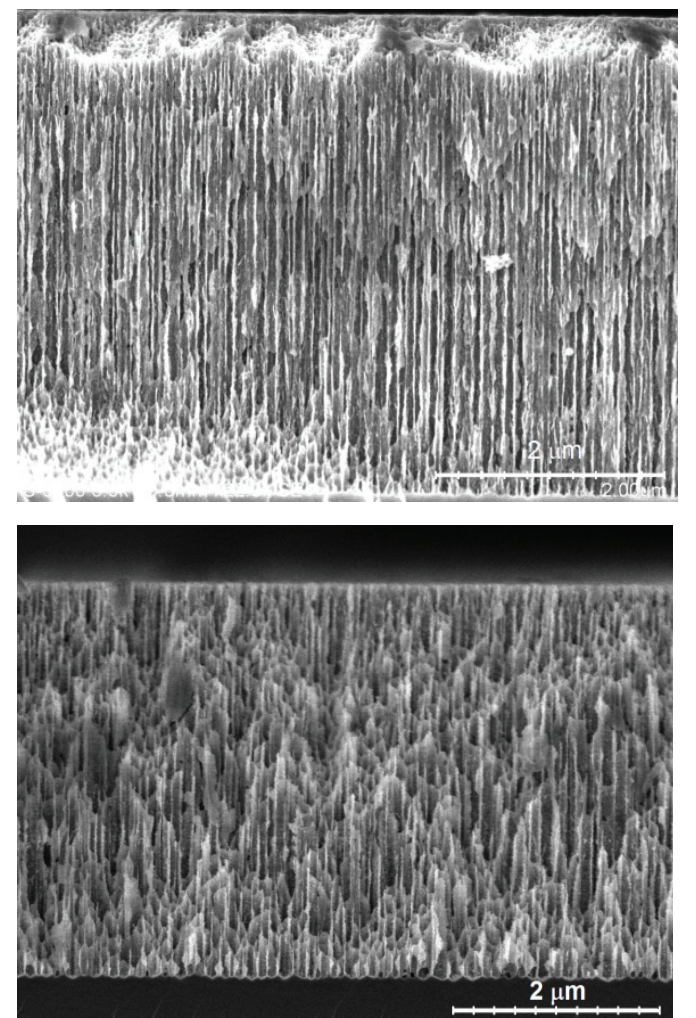

b)

d)

Figura 2. Micrografías de estructuras de SiP; Monocapa fabricada con 55 mA, a) vista superficial (inset: amplificación a 200,000 aumentos, escala $200 \mathrm{~nm}$ ), b) sección transversal; monocapa fabricada con 65 mA, c) vista superficial (inset: amplificación a 200,000 aumentos, escala $200 \mathrm{~nm}$ ), d) sección transversal 
una corriente de $65 \mathrm{~mA}$ mostrada en la figura 2c, donde se observa un diámetro de poro de $50.519 \pm 12.9705 \mathrm{~nm}$. Ambas monocapas poseen una superficie tipo esponja con un poro no circular. La sección transversal de las monocapas se muestran en las figuras $2 \mathrm{~b}$ y d correspondientes a la monocapa de $55 \mathrm{~mA}$ con un espesor de $\sim 4.20 \mu \mathrm{m}$ y a la monocapa de $65 \mathrm{~mA}$ con espesor de $\sim 3.61 \mu \mathrm{m}$, respectivamente. Comparando la sección trasversal de ambas monocapas, se observan los canales formados en la formación del poro y la diferencia de tamaño de dichos canales entre ambas estructuras.

Si bien, el mecanismo de formación de poro se encuentra en discusión aún, se sabe que la disolución del silicio requiere presencia de iones $\left(F^{-}\right)$y huecos $\left(h^{+}\right)$ para silicio tipo p. Cuando los iones fluoruros se entregan más rápido que los huecos, la región entre los poros del SiP se agota de huecos y el ataque se produce solo en las puntas de poro, donde los huecos se enfocan por un campo eléctrico. Cuando la densidad de corriente decrece, el número de huecos en la punta del poro decrece, lo que ocasiona diámetros de poros más pequeños (Ouyang et al., 2005).

\section{ESPECTROSCOPIA UV-VIS-NIR CON REFLECTANCIA ESPECULAR}

Se registró el espectro de reflectancia (Perkin Elmer UV-Vis-NIR WinLab Lambda 900) de las monocapas de SiP en cada paso de la funcionalización. En la figura 3 se observa el espectro de reflectancia (izquierda) y su respectivo pico característico del EOE (derecha), tomados de una monocapa de SiP fabricada con una corriente de $65 \mathrm{~mA}$ por $3 \mathrm{~min}$. Las etiquetas de la parte superior izquierda de los interferogramas denotan el paso de la funcionalización después de que se tomó el espectro. Las líneas punteadas en las gráficas permiten observar cómo a cada etapa existe un movimiento o "shift" en el espectro de reflectancia y, por lo tanto, en el pico de EOE. Esto comprueba la modificación superficial de las paredes del SiP por efecto de la inmovilización de moléculas.
En la tabla 1 se comparó el cambio de EOE en las diferentes monocapas de SiP y la concentración de AChE en la solución de impregnación de la última etapa del proceso de funcionalización. La muestra F65-01 que posee el mayor diámetro de poro y se incubó en una solución de $0.2 \mathrm{mg} / \mathrm{ml}$ de AChE, mostró el mayor desplazamiento del EOE. Para el mismo tipo de estructura porosa (F65-02), pero usando una solución de incubación de $0.1 \mathrm{mg} / \mathrm{ml}$ el desplazamiento disminuyó. Por otro lado, las muestras sintetizadas a 55 mA, F55-01 y F55-02, mostraron un desplazamiento menor al obtenido por sus contrapartes de mayor tamaño de poro a la misma concentración del AChE en la solución de impregnación. Al reducir el tamaño del poro es evidente que se requiere una mayor concentración de AChE en

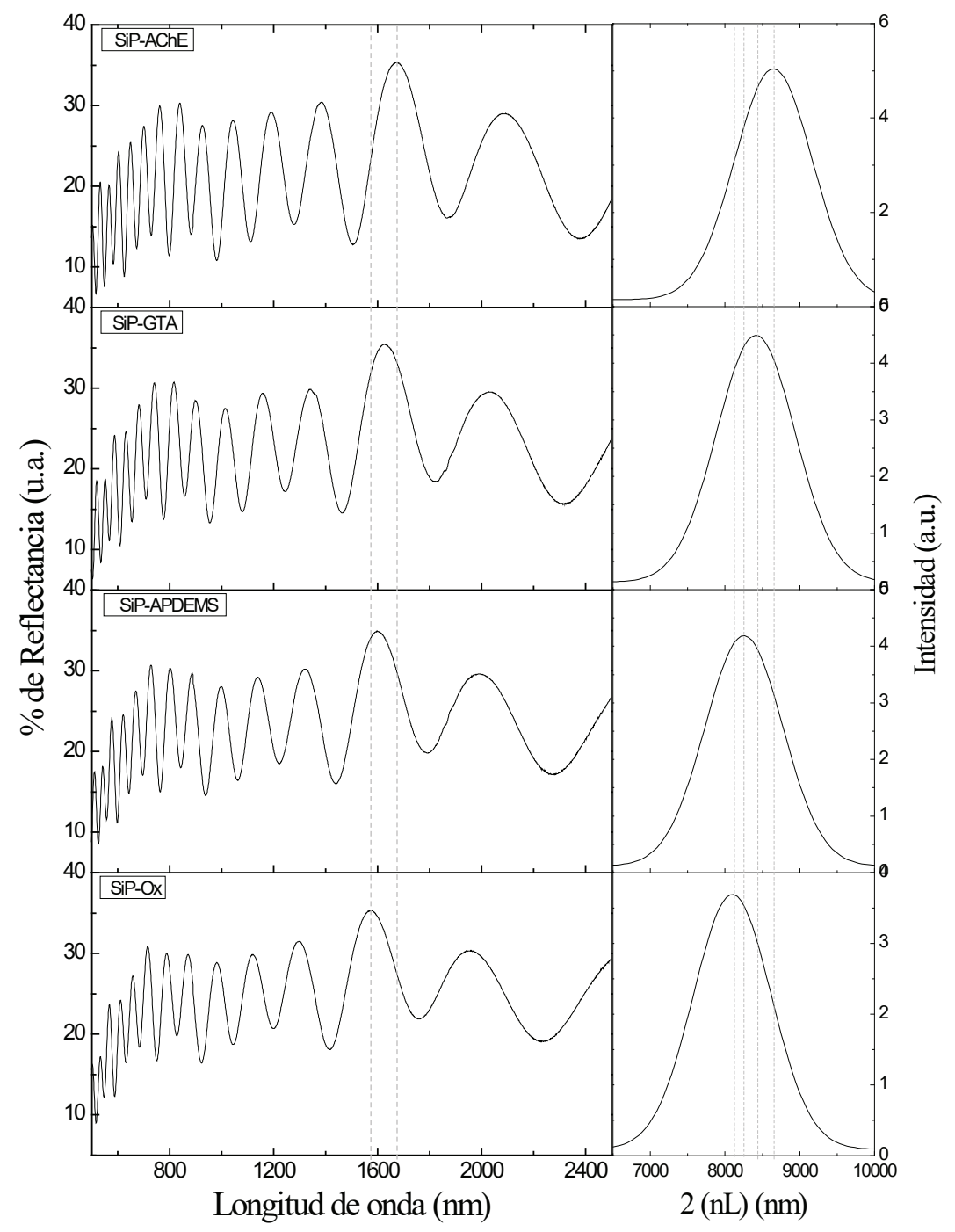

Figura 3. Espectros de reflenctancia y picos del EOE de una monocopa de SiP fabricada con una corriente de $65 \mathrm{~mA}$ por 3 min, tomados a cada paso del proceso de funcionalización con AChE 
Tabla 1. Dependencia del cambio del EOE en las monoacapas de SiP a la corriente de fabricación y a la concentración de AChE de la solución de impregnación

\begin{tabular}{ccccc}
\hline Muestra & Corriente $(\mathrm{mA})$ & $\begin{array}{c}\text { Tiempo de } \\
\text { anodizado }\end{array}$ & $\begin{array}{c}\text { Concentración de AChE en la solución } \\
\text { de impregnación }(\mathrm{mg} / \mathrm{ml})\end{array}$ & $\Delta$ EOE $(\mathrm{nm})$ \\
\hline F55-01 & 55 & 240 & 0.2 & 160 \\
F55-02 & 55 & 240 & 0.1 & 0 \\
F65-01 & 65 & 180 & 0.2 & 212 \\
F65-02 & 65 & 180 & 0.1 & 150 \\
\hline
\end{tabular}

la solución de incubación para mostrar un $\triangle \mathrm{EOE}$. Es importante resaltar que un menor tamaño de poro admite menos infiltración de material orgánico (Palestino et al., 2009), por lo que para generar un desplazamiento de EOE se requiere una mayor concentración de la enzima en la solución de impregnación. En la monocapa de mayor diámetro de poro la concentración de $0.2 \mathrm{mg} /$ $\mathrm{ml}$ de $\mathrm{AChE}$ en la solución de impregnación generó mayor $\triangle \mathrm{EOE}$, por lo tanto, un mayor enlazamiento de la proteína sobre las paredes del poro, por lo que estas condiciones de preparación se eligieron para los estudios subsecuentes.

\section{ESPECTROSCOPIA DE FTIR}

El espectro de infrarrojo con transformada de Fourier (Varian 660 IR) se registró después de la funcionalización con GTA y con AChE (figura 4) lo que permitió monitorear la formación de los enlaces generados en estos dos pasos del proceso de impregnación con AChE. La gráfica alojada dentro de la figura 4 muestra el espectro de IR tomado desde $800-4.000 \mathrm{~cm}^{-1}$, en ella se observan los dos picos más prominentes en ambos espectros (SiP/GTA y SiP/AChE) a 1.054 y $1.183 \mathrm{~cm}^{-1}$ que corresponden al modo óptico transversal (TO) y al modo óptico longitudinal (LO) de los enlaces $\mathrm{Si}-\mathrm{O}-\mathrm{Si}$, respectivamente; estos picos se generan debido al proceso de oxidación térmica. El pico a $801 \mathrm{~cm}^{-1}$ se asigna a los grupos funcionales de Si-OH. El gráfico principal muestra los picos resultantes de la funcionalización con GTA y AChE. La línea sólida pertenece al espectro de la muestra de SiP/GTA y se observan los picos de las vibraciones de deformación de $\mathrm{CH}_{2}$ y estiramiento del $\mathrm{C}=\mathrm{O}$ en 1.410 y $1.724 \mathrm{~cm}^{-1}$, respectivamente. Además el pico a $1.647 \mathrm{~cm}^{-1}$ se asigna al modo de vibración de $\mathrm{N}=\mathrm{C}$ que envuelve la formación de los enlaces entre las moléculas de la superficie aminosilanizada y el GTA. Los grupos alifáticos inmovilizados en la superficie se observan como los picos en 2.864 y $2.938 \mathrm{~cm}^{-1}$ que pertenecen a los modos de vibración asimétrico y simétrico de los enlaces C-H. Después de la inmovilización de la proteína (línea punteada) se observa la aparición de dos picos significativos a 1.540 y $1.651 \mathrm{~cm}^{-1}$ atribuidos a la banda amida II (vibración de $\mathrm{N}-\mathrm{H})$ y amida I $(\mathrm{C}=\mathrm{O})$ que están relacionadas con la proteína. Otro pico apenas visible se observa por efecto de la inmovilización de la proteína a $3.071 \mathrm{~cm}^{-1}$ que se asocia al modo de estiramiento del enlace $\mathrm{N}-\mathrm{H}$. La designación de picos se realizó tomando como referencia el estudio previo de la funcionalización biológica, realizado por Hiraoui et al. (2011).

\section{ACTIVIDAD ENZIMÁTICA}

$\mathrm{Al}$ aplicar la FFT al espectro de reflectancia (\%R vs $1 / \lambda)$ de una monocopa de $\mathrm{SiP}$, se obtiene un pico característico, donde el punto más alto representa el EOE con cierta amplitud. La amplitud de un pico, $A$, en la FFT del espectro está relacionado con las interfaces limitantes de la capa porosa según la ecuación

$A=k \rho_{a} \rho_{b}$

donde

$k=$ constante de proporcionalidad

$\rho_{a}$ y $\rho_{b}=$ contraste entre los índices de refracción en cada interface como se describe en la ecuación siguiente

$\rho a=\frac{n_{\text {med }}-n_{1}}{n_{\text {med }}+n_{1}}, \rho_{b}=\frac{n_{1}-n_{S i}}{n_{1}+n_{S i}}$

donde $n_{\text {medr }} n_{1}, n_{S i}$ constituyen el índice de refracción de la solución, la capa porosa y el silicio en bulto, respectivamente. La cantidad $n_{1}$ representa el índice de refracción de todo lo que contiene esa capa (silicio, oxido de silicio, solución, biomoleculas, etcétera). Si bien, los modelos para ajustar los datos con el resultado de la FFT son bastantes simplificados, estos proporcionan una imagen coherente de las propiedades ópticas de las nanoestructuras (Pacholski et al., 2005). Al usar el RIFTS como método de seguimiento de la actividad enzimática de la superficie de SiP/AChE se observó una mayor respuesta en la señal de amplitud. El cambio en la magnitud de la amplitud demostró ser efectivo y sensible en la detección de cambios relativos a la interface SiPsolución (Balderas et al., 2014). El cambio de amplitud 


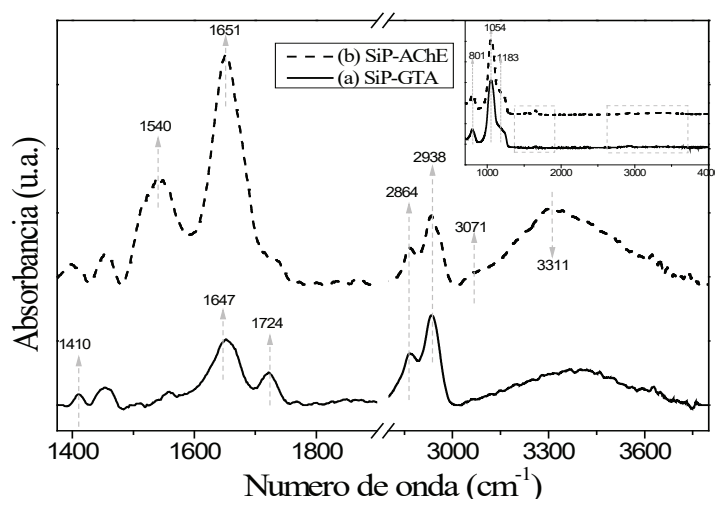

Figura 4. Espectro de FTIR de las superficies tipo SiP/GTA (línea solida) y SiP/AChE (línea punteada). El gráfico insertado muestra el espectro completo de las muestras desde 800 a $4000 \mathrm{~cm}^{-1}$

respecto a la amplitud inicial $\left(\Delta A=A-A_{0}\right)$ se registró como señal para dar seguimiento a la actividad enzimática. En la figura 5 se observa el incremento de la amplitud del pico de la FFT debido al avance de la reacción al usar la solución 1. Esta muestra se sometió a reacción en tres ocasiones, por lo que se demuestra la posibilidad del re-usar el sustrato modificado, aunque se observó una pérdida de la actividad catalítica la tercera vez que se hizo reaccionar.

Cuando el SiP/AChE reacciona en presencia del As (III) se observa un cambio en la respuesta del cambio de amplitud atribuido a la inhibición de la AChE. En la figura 6 se muestra el resultado de los diferentes ensayos enzimáticos llevados a cabo en la celda flujo, usando la solución 2. Sin la presencia de As (III) el $\Delta A$ alcanza valores 1.1 unidades, sin embargo, se observa que al aumentar la concentración de As (III) en la solución reactiva los valores de $\Delta A$ disminuyen. Se observó que a concentraciones de As (III) mayores de $\sim 30 \mu \mathrm{M}$ no se

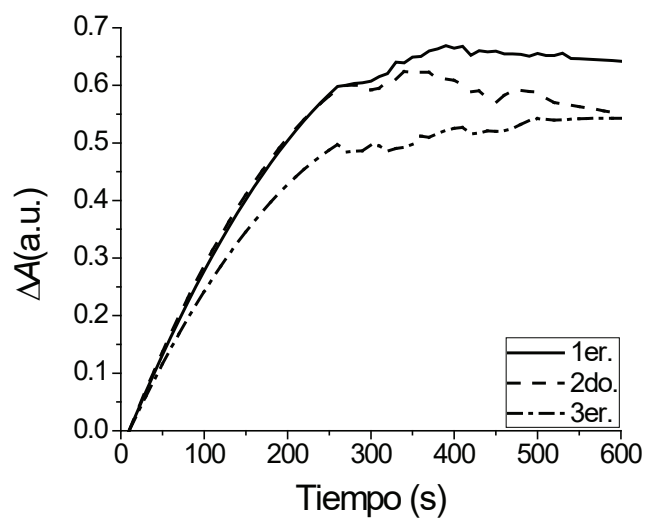

Figura 5. Respuesta del ensayo enzimático del SiP/AChE usando la solución 1. El sustrato de funcionalizado se sometió a reacción en 3 ocasiones consecutivas

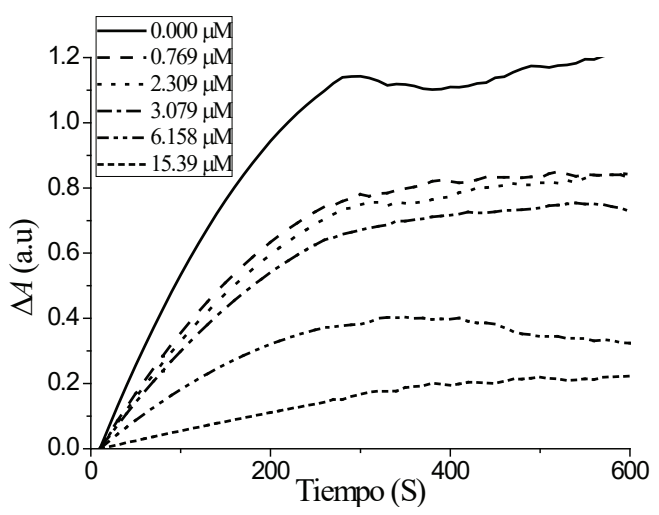

Figura 6. Respuesta del $\Delta A$ del ensayo enzimático del SiP/AChE a diferentes concentraciones de As (III)

presentó actividad enzimática, mientras que a valores menores $\sim 0.77 \mu \mathrm{M}$ la actividad catalítica se compara con la que se ilustra sin la presencia de As (III), por lo que este valor se considera como el límite de detección.

\section{ConClusiones}

La carga de enzima en la estructura de SiP depende de la concentración inicial de la solución de impregnación de AChE, así como del tamaño de poro del sustrato nanoestructurado, por lo que un mayor tamaño de poro permite una mayor infiltración de la solución de impregnación de la proteína. La inmovilización de la AChE sobre el SiP se realizó mediante la formación de enlaces covalentes del tipo amida I y amida II. En ausencia de As (III) los sustratos de SiP/AChE pueden reutilizarse para propósitos de detección en cierta medida. El sistema de detección propuesto en este trabajo de investigación demostró ser efectivo para la detección de As (III) con un límite de detección de $\sim 0.77 \mu \mathrm{M}$.

\section{Agradecimientos}

Este trabajo se financió por el CONACyT. Proyecto CIAM Núm. 188657.

\section{RefERENCIAS}

Aarsland D., Mosimann U.P., McKeith I.G. Role of cholinesterase inhibitors in Parkinson's disease and dementia with Lewy bodies. Journal of Geriatric Psychiatry and Neurology, volumen 17 (número 3), 2004: 164-171.

Balderas-Valadez R.F., Weiler M., Agarwal V. Pacholski C. Optical characterization of porous silicon monolayers decorated with hydrogel microspheres. Nanoscale Research Letters, volumen 9 (número 1), 2014: 1-7. 
Balderas-Valadez R.F., Agarwal V. Porous silicon functionalization for possible arsenic adsorption. Nanoscale Research Letters, volumen 9 (número 1), 2014: 1-6.

De Mora K., Joshi N., Balint B.L., Ward F.B., Elfick A, French C.E. A $\mathrm{pH}$-based biosensor for detection of arsenic in drinking water. Analytical and Bioanalytical chemistry, volumen 400 (número 4), 2011: 1031-1039.

Diesel E., Schreiber M., Van der Meer J.R. Development of bacteria-based bioassays for arsenic detection in natural waters. Analytical and Bioanalytical Chemistry, volumen 394 (número 3), 2009: 687-693.

Ellman G.L., Courtney K.D., Andres V., Featherstone R.M. A new and rapid colorimetric determination of acetylcholinesterase activity. Biochemical Pharmacology, volume 7 (número 2), 1961: 88-95.

Hiraoui M., Guendouz M., Lorrain N., Moadhen A., Haji L. Oueslati M. Spectroscopy studies of functionalized oxidized porous silicon surface for biosensing applications. Materials Chemistry and Physics, volumen 128 (número 1), 2011: 151-156.

Jane A., Dronov R., Hodges A., Voelcker N.H. Porous silicon biosensors on the advance. Trends in biotechnology, volume 27 (número 4), 2009: 230-239.

Jane A., Dronov R., Hodges A., Voelcker N.H. Porous silicon biosensors on the advance. Trends in Biotechnology, volumen 27 (número 4), 2009: 230-239.

Liu Q., Peng H., Lu X., Le X.C. Enzyme-assisted extraction and liquid chromatography mass spectrometry for the determination of arsenic species in chicken meat. Analytica Chimica Acta, volumen 888, 2015: 1-9

Li J., Chen L., Lou T., Wang Y. Highly sensitive SERS detection of As3+ ions in aqueous media using glutathione functionalized silver nanoparticles. ACS Applied Materials \& Interfaces, volumen 3 (número 10), 2001: 3936-3941.

Maher W.A., Ellwood M.J., Krikowa F., Raber G., Foster S. Measurement of arsenic species in environmental, biological fluids and food samples by HPLC-ICPMS and HPLC-HG-AFS. Journal of Analytical Atomic Spectrometry, volumen 30 (número 10), 2015: 2129-2183.

Moss D.E., Perez R.G., Kobayashi H. Cholinesterase Inhibitor Therapy in Alzheimer's Disease: The Limits and Tolerability of Irreversible CNS-Selective Acetylcholinesterase Inhibition in Primates. Journal of Alzheimer's Disease, volumen 55 (número 3), 2016, 1285-1294.

Orosco M.M., Pacholski C., Sailor M.J. Real-time monitoring of enzyme activity in a mesoporous silicon double layer. Nature Nanotechnology, volumen 4 (número 4), 2009: 255-258.

Ouyang H. y Fauchet P.M. Biosensing using porous silicon photonic bandgap structures, en: Optics East 2005, International Society for Optics and Photonics, 600508-600508.

Pacholski C., Sartor M., Sailor M.J., Cunin F., Miskelly G.M. Biosensing using porous silicon double-layer interferometers: reflective interferometric Fourier transform spectroscopy. Journal of the American Chemical Society, volume 127 (número 33), 2005: 11636-11645.
Pacholski C., Yu C., Miskelly G.M., Godin D., Sailor M.J. Reflective interferometric fourier transform spectroscopy: a self-compensating label-free immunosensor using double-layers of porous SiO2. Journal of the American Chemical Society, volumen 128 (número 13), 2006, 4250-4252.

Pacholski C., Perelman L.A., VanNieuwenhze M.S., Sailor M.J. Small molecule detection by reflective interferometric Fourier transform spectroscopy (RIFTS). Physica Status Solidi (a), volume 206 (número 6), 2009: 1318-1321.

Palestino G., Martin M., Agarwal V., Legros R., Cloitre T., Zimányi L., Gergely C. Detection and light enhancement of glucose oxidase adsorbed on porous silicon microcavities. Physica Status Solidi (c), volumen 6 (número 7), 2009: 1624-1628.

Pradhan M., Maji S., Sinha A.K., Dutta, S., Pal T. Sensing trace arsenate by surface enhanced Raman scattering using a FeOOH doped dendritic Ag nanostructure. Journal of Materials Chemistry A, volumen 3 (número 19), 2015: 10254-10257.

Sailor M.J. Porous silicon in practice, Weinheim, Wiley-VCH, 2012.

Salomons W., Förstner U., Mader P. Heavy metals: problems and solutions, Springer Science \& Business Media, 2012.

Sanllorente-Méndez S., Domínguez-Renedo O., Arcos-Martínez M.J. Immobilization of acetylcholinesterase on screen-printed electrodes. Application to the determination of arsenic (III). Sensors, volumen 10 (número 3), 2010: 2119-2128.

Sarkar P., Banerjee S., Bhattacharyay D., Turner A.P. Electrochemical sensing systems for arsenate estimation by oxidation of L-cysteine. Ecotoxicology and Environmental Safety, volumen 73 (número 6), 2010: 1495-1501.

Shtenberg G., Massad-Ivanir N., Segal E. Detection of trace heavy metal ions in water by nanostructured porous Si biosensors. Analyst, volumen 140 (número 13), 2015: 4507-4514.

Stoytcheva M., Sharkova V., Panayotova M. Electrochemical approach in studying the inhibition of acetylcholinesterase by arsenate (III): analytical characterisation and application for arsenic determination. Analytica Chimica Acta, volumen 364 (número 1), 1998: 195-201.

Vincent G. Optical properties of porous silicon superlattices. Applied Physics Letters, volume 64 (número 18), 1998: 2367-2369.

Wang X., Xu G., Chen P., Liu X., Fang Y., Yang S., Wang G. Arsenic speciation analysis in environmental water, sediment and soil samples by magnetic ionic liquid-based air-assisted liquid-liquid microextraction. RSC Advances, volumen 6 (número 111), 2016: 110247-110254.

Whitacre D.M. Reviews of environmental contamination and toxicology, Springer, 2012.

Zhuravlev A., Zacharia A., Gucer S., Chebotarev A., Arabadji M., Dobrynin A. Direct atomic absorption spectrometry determination of arsenic, cadmium, copper, manganese, lead and zinc in vegetable oil and fat samples with graphite filter furnace atomizer. Journal of Food Composition and Analysis, volumen 38, 2015: 62-68. 


\section{Citación sugerida:}

\section{Citación estilo Chicago}

Balderas-Valadez, Ruth Fabiola, Vivechana Agarwal. Uso del silicio poroso funcionalizado con acetilcolinesterasa como plataforma de detección de arsénico (III). Ingeniería Investigación y Tecnología, XVIII, 03 (2017): 321-329.

\section{Citación estilo ISO 690}

Balderas-Valadez R.F., Agarwal V. Uso del silicio poroso funcionalizado con acetilcolinesterasa como plataforma de detección de arsénico (III). Ingeniería Investigación y Tecnología, volumen XVIII (número 3), julio-septiembre 2017: 321-329.

\section{SemblanZas de los autores}

Ruth Fabiola Balderas-Valadez. Obtuvo su maestría en ingeniería química por la Universidad Autónoma de San Luis Potosí y su doctorado en ingeniería y ciencias aplicadas por la Universidad Autónoma del Estado de Morelos (2016), es candidata al Sistema Nacional de Investigadores, actualmente es posdoctorante en la Benemérita Universidad Autónoma de Puebla. Sus líneas de investigación son síntesis y análisis de materiales semiconductores y desarrollo de estructuras fotónicas.

Vivechana Agarwal. Obtuvo su maestría en física y su doctorado en física de materiales por la Universidad de Delhi, India (1999). Actualmente es profesor investigador titular " $\mathrm{C}$ " del Centro de Investigación en Ingeniería y Ciencias Aplicadas de la UAEM, con nivel 9 en estímulos al desempeño del personal docente; es miembro del Sistema Nacional de Investigadores nivel III. Sus contribuciones científicas van desde la investigación básica hasta el desarrollo tecnológico en los ámbitos de síntesis, caracterización y aplicaciones del silicio poroso y óxidos metálicos, diseño y fabricación de estructuras fotónicas, nanocompuestos y aplicaciones de sensado. 
DOI: http://dx.doi.org/10.22201/fi.25940732e.2017.18n3.028 